\section{His bundle pacing in heart failure: $A$ review of current literature}

\author{
Rahul Chaudhary ${ }^{1 *}$ and Jalaj Garg ${ }^{2}$ \\ ${ }^{1}$ Division of Hospital Internal Medicine, Mayo Clinic, Rochester, MN, USA \\ ${ }^{2}$ Division of Cardiology, Medical College of Wisconsin, Milwaukie, WI, USA
}

\section{Abstract}

Biventricular (BiV) pacing revolutionized the heart failure management in patients with sinus rhythm and left bundle branch block; however, left ventricular-lead placement is not always technically possible. Also, BiV pacing does not fully normalize ventricular activation and, therefore, the ventricular resynchronization is imperfect. On the other hand, right ventricular pacing for bradycardia may cause or worsen heart failure in some patients by causing dyssynchronous ventricular activation. His bundle pacing comes as an alternative to current approaches as it activates the ventricles via the native His-Purkinje system, resulting in true physiological pacing, and, therefore, is a promising site for pacing in bradycardia and traditional CRT indications in cases where it can overcome left bundle branch block. Furthermore, it has the potential to open up new indications for pacing therapy in heart failure, such as targeting patients with PR prolongation, but a narrow QRS duration. In this article we explore the history, clinical evidence, proposed mechanisms, procedural characteristics, and the role in current therapy of His bundle pacing in the prevention and treatment of heart failure.

\section{More Information}

*Address for Correspondence: Rahul Chaudhary MD FACP, Associate Consultant, Clinical Assistant Professor of Medicine, Division of Hospital Internal Medicine, Department of Internal Medicine, Mayo Clinic, 200 First Street SW, Rochester, MN 55905, USA, Tel: 507-255-8043; Fax: 507-255-9189; Email: Chaudhary.rahul@mayo.edu

Submitted: 10 January, 2020

Approved: 02 March 2020

Published: 03 March 2020

How to cite this article: Chaudhary R, Garg J. His bundle pacing in heart failure: A review of current literature. J Cardiol Cardiovasc Med. 2020; 5: 042-046.

\section{DOI: 10.29328/journal.jccm.1001084}

Copyright: @ 2020 Chaudhary R, et al. This is an open access article distributed under the Creative Commons Attribution License, which permits unrestricted use, distribution, and reproduction in any medium, provided the original work is properly cited.

Keywords: His bundle; Arrhythmia; Heart failure

Check for updates

OPEN ACCESS

\section{History of his bundle}

The first anatomical description of His bundle was made by Wilhelm His Jr. in 1893. He was the first to describe the significance of His bundle as a conduit to conduction between the auricles to ventricular septal wall. The first physiological readings of His bundle were only recorded in 1958 by Alanis, et al. [1]. In 1959, Giraud, et al. performed the first human recordings of His bundle in patients with tetralogy of Fallot [2]. Subsequently, numerous observations and clinical trials further elucidated the role of His bundle in the normal cardiac conduction system paving way for its therapeutic utility in pathological states like heart failure.

\section{Anatomy of his bundle}

His bundle originates from the atrioventricular (AV) node located at the inferior border of Koch's triangle. His bundle then extends anterosuperiorly into the central fibrous body and traverses through the membranous septum. It then divides into right and left bundle branches (Figure 1). There are 3 different types of anatomic variations of His bundle described by Kawashima and Sasaki [3]. The most common anatomy observed was type I in $46.7 \%$ of specimens where the His bundle was covered with a thin layer of myocardial fiber as it traverses along the lower border of the membranous septum. The second most common anatomical variation observed was type II pattern which has the deepest course underneath a thick myocardial layer, traversing inferior to the membranous septum. Type III His bundle was seen in $21 \%$ of specimens and was with no insulation and the thinnest myocardial depth. The differing depths of the His bundle likely account for the cases with an obvious His bundle injury current during lead deployment and low capture thresholds in some patients.

Anatomy within the His bundle was further demonstrated to be composed of longitudinally oriented Purkinje strands divided by collagen sheaths believed to provide electrical insulation. Several studies showed that pacing at His bundle site was successful in $85 \%$ of patients with high-grade AV

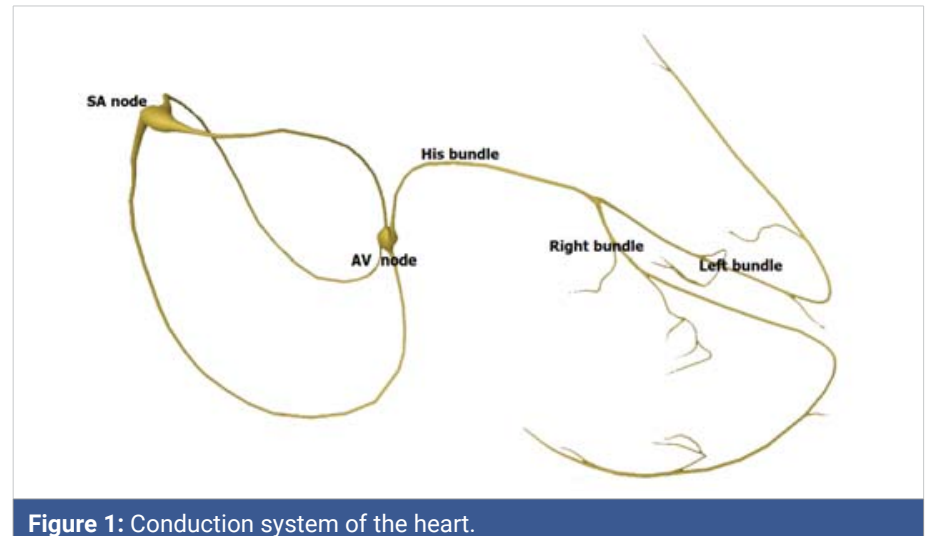


blocks [4]. Furthermore, studies showing the correction of bundle branch blocks with distal pacing at His bundle promoted the concept of longitudinal dissociation suggesting that individual fibers within the common His bundle are predestined for the bundle branches [5,6]. This concept was elegantly demonstrated by Narula, in 1977 [6]. Patients with left bundle branch block (LBBB) and baseline prolonged $\mathrm{HV}$ intervals were studied and pacing slightly distal to the proximal His bundle resulted in narrowing of the QRS. ElSherif, et al. published both clinical and experimental evidence for longitudinal dissociation in the diseased His bundle in humans in 1978 [7].

\section{Heart failure and his bundle}

The patients with heart failure (due to ischemic or non-ischemic etiology) commonly have regions of delayed myocardial activation and contraction, leading to cardiac dyssynchrony. The dyssynchrony manifests itself in the electrocardiogram as prolongation of QRS interval to more than $120 \mathrm{~ms}$. Approximately one-third of the heart failure patients have progressive prolongation of QRS duration which been associated with poor clinical outcomes [8]. These observations were further confirmed when patients with left ventricular systolic dysfunction, cardiac dyssynchrony, and symptomatic heart failure who underwent cardiac resynchronization therapy (CRT) were shown to have improvement in ventricular contractile function, reversal of ventricular remodeling, a sustained improvement in left ventricular ejection fraction (LVEF) and reduction in rehospitalizations, symptoms, and risk of death with improved quality of life [9-11]. The current heart failure guidelines recommend use of CRT in patients who have LVEF of $\leq 35 \%$, sinus rhythm and (a) LBBB with QRS duration of $\geq 150$ ms and New York Heart Association (NYHA) class II, III, or ambulatory IV symptoms on guideline-directed medical therapy (GDMT) (Class I); (b) LBBB with a QRS duration of 120 to $149 \mathrm{~ms}$ and NYHA class II, III, or ambulatory IV symptoms on GDMT (Class IIa); (c) a non-LBBB pattern with a QRS duration of $\geq 150 \mathrm{~ms}$ and NYHA class III/ambulatory class IV symptoms on GDMT (Class IIa); (d) non-LBBB pattern with a QRS duration of $\geq 150 \mathrm{~ms}$, and NYHA class II symptoms on GDMT (Class IIb); (e) a non-LBBB pattern with QRS duration of 120 to $149 \mathrm{~ms}$, and NYHA class III/ambulatory class IV on GDMT (Class IIb); (f) a non-LBBB pattern with a QRS duration of $\geq 150 \mathrm{~ms}$, and NYHA class II symptoms on GDMT (Class IIb) (Table 1) [12]. Additionally, CRT has been recommended for patients with LVEF of $\leq 35 \%$ on GDMT and (i) atrial fibrillation if the patient requires ventricular pacing or otherwise meets CRT criteria and in atrioventricular nodal ablation or pharmacological rate control will allow near $100 \%$ ventricular pacing with CRT (Class IIa); (ii) patients undergoing placement of a new or replacement device implantation with anticipated requirement for significant (> 40\%) ventricular pacing (Class IIa) [12] (Table 1). The success of CRT in this patient population has been attributed to the atrial-synchronized biventricular pacing leading to an improved coordination between right and left ventricular contraction. The traditional approach for CRT is through biventricular pacing with right ventricular apical lead and a LV lead placed through the lateral branches of the coronary sinus. However, CRT is not without its limitations. Indeed, several studies have shown that up to onethird of patients treated with CRT do not derive any clinical or echocardiographic benefit and instead, may worsen $[9,10,13]$. Several mechanisms for the suboptimal response of CRT have been identified including baseline QRS morphology/duration, diminished CRT due to arrhythmias, suboptimal location of LV lead, LV latency, and suboptimal AV and VV timing [14-16]. Another major limitation of biventricular pacing in CRT is failure of LV lead deployment due to limitations in coronary sinus anatomy, and the standard of care suggests epicardial LV lead insertion as the next alternative. Conventional CRT has also shown a lack of benefit in patients with a normal QRS duration and among patients with RBBB [17]. It is also well known that long-term RV pacing can worsen LV function and heart failure.

To overcome some of the limitations observed with CRT, permanent His-bundle pacing (PHBP) has recently been explored as an alternative to deliver physiological ventricular pacing. Direct stimulation of the His bundle has been proposed to be the most physiologic mode of ventricular pacing [18]. The physiologic benefit of PHBP is the ability to utilize intrinsic His-purkinje system to stimulate the ventricles resulting in synchronous and a more physiologic electrical and mechanical activation. Additionally, PHBP can be used as a bail-out strategy in cases patients with limitations of coronary venous anatomy for LV lead placement providing

Table 1: Indications of cardiac resynchronization therapy

Indications of cardiac resynchronization therapy as per heart failure guidelines

Among patients with left ventricular ejection fraction (LVEF) $\leq 35 \%$ on goal-directed medical therapy and:

Class I recommendations Left bundle branch block (LBBB) with QRS duration of $\geq 150 \mathrm{~ms}$, and New York Heart Association (NYHA) class II, III, or ambulatory IV symptoms in sinus rhythm LBBB with a QRS duration of 120 to $149 \mathrm{~ms}$ and NYHA class II, III, or ambulatory IV symptoms in sinus rhythm non-LBBB pattern with a QRS duration of $\geq 150 \mathrm{~ms}$ and NYHA class III/ambulatory class IV symptoms on GDMT

Class Ila recommendations In atrial fibrillation if the patient requires ventricular pacing or otherwise meets CRT criteria and in atrioventricular nodal ablation or pharmacological rate control will allow near $100 \%$ ventricular pacing with CRT

undergoing placement of a new or replacement device implantation with anticipated requirement for significant (>40\%) ventricular pacing non-LBBB pattern with a QRS duration of $\geq 150 \mathrm{~ms}$, and NYHA class II symptoms in sinus rhythm non-LBBB pattern with QRS duration of 120 to $149 \mathrm{~ms}$, and NYHA class III/ambulatory class IV in sinus rhythm non-LBBB pattern with a QRS duration of $\geq 150 \mathrm{~ms}$, and NYHA class II symptoms in sinus rhythm 
a minimally invasive alternative to an open chest procedure for epicardial lead placment. Other advantages include the lack of potential complications from LV lead placement including coronary sinus dissection, venous perforation, cardiac tamponade, overstimulation of phrenic nerve and the potential for proarrhythmia [19].

PHBP came to limelight in the year 2000, after Deshmukh, et al. published the paper on safety and feasibility of PHBP in 18 patients with chronic atrial fibrillation, $\mathrm{LVEF}<40 \%$, QRS $<$ $120 \mathrm{~ms}$ and NYHA class III-IV HF symptoms who underwent AV nodal ablation [20]. They were successful in achieving PHBP in two thirds of their patients and demonstrated positive LV remodeling and improvement in LVEF (from 20 $\pm 9 \%$ to $31 \pm 11 \%, p<0.01$ ) in this cohort. This trial showed $\mathrm{PHBP}$ to be a viable alternative to $\mathrm{RV}$ pacing.

\section{Clinical evidence comparing HBP with CRT for heart failure}

There are limited data on PHBP as an alternative to biventricular pacing for CRT. The available studies have reported the use of PHBP for CRT with success rate ranging from $56 \%$ to $92 \%$. The baseline characteristics of patient population for the following trials is elaborated in table 2. Barba-Pichardo, et al. reported their experience with PHBP in 16 patients with cardiomyopathy and failed CRT [21]. Successful CRT by PHBP was obtained in 9 patients, corresponding to a success rate of $56 \%$ of the initially proposed patients for CRT. Mean QRS duration reduced from $166 \pm 8 \mathrm{~ms}$ to $97 \pm 9 \mathrm{~ms}(p<0.01)$. HBP threshold at implant $3.09 \pm 0.44 \mathrm{~V}$ at $1 \mathrm{~ms}$ and at follow-up was $3.70 \pm 0.54 \mathrm{~V}(p<$ $0.05)$. NYHA functional class improved from class III to class II and there was an improvement in LVEF (from $29.00 \pm 0.05 \%$ to $36.00 \pm 0.05 \%, p<0.05)$ and LV dimensions $(p<0.05)$.

Lustgarten, et al. compared HBP versus biventricular pacing in a randomized crossover patient-blinded study in 29 patients with indications for CRT defibrillator implants [22]. All patients received both a coronary sinus LV lead and an HBP lead connected to the LV port via a Y-adapter. After an initial assignment, patients were crossed over to the other group after 6 months and followed for another 6 months. The study reported successful electrical resynchronization in $21(72 \%)$ cases with HBP for CRT. At the end of 1 year of study, 12 patients completed the crossover analysis. Both groups of patients demonstrated significant improvements in 6-min walk, NYHA functional class, quality of life and LVEF. They concluded that HBP to have an equivalent CRT response compared to conventional BiV pacing.

$\mathrm{Su}$, et al. demonstrated the feasibility of incorporating HBP into a CRT-D/ICD system by comparing various pacing configurations in 16 patients who underwent successful CRT-D with HBP lead in the LV port and 13 dual chamber ICD implants (patients with permanent AF) with the HBP lead in atrial port [23]. Additionally, they observed that capture thresholds and R-wave sensing can be optimized using an integrated bipolar configuration with the RV lead. In another study, Vijayaraman et al. showed successful HBP in $90.6 \%$ patients $(n=32), 14$ of whom had failed coronary sinus leads and two non-responders to biventricular CRT. They observed QRS narrowing, improved LVEF and NYHA functional class reiterating that HBP in lieu of an LV lead is also feasible [24]. Ajijola, et al. evaluated 21 patients with indication for CRT implant to incorporate a His-bundle lead for CRT in lieu of a coronary sinus lead [25]. HBP implantation was successful in $76 \%$ patients (16 out of 21 patients) with significant narrowing of the QRS duration (from $180 \pm 23 \mathrm{~ms}$ to $129 \pm$ $13 \mathrm{~ms}, p<0.0001$ ), NYHA class (from Class III to Class II, $p$ $<0.001$ ), LVEF (from $27 \pm 10 \%$ to $41 \pm 13 \%, p<0.001$ ) and $\mathrm{LV}$ internal dimension in diastole (from $5.4 \pm 0.4 \mathrm{~cm}$ to 4.5 $\pm 0.3 \mathrm{~cm}, p<0.001$ ) at 6 -month follow-up. In a recent study, Sharma et al. assessed 106 patients with CRT indications for HBP as a rescue strategy for failed LV lead or nonresponse to biventricular pacing, or as a primary strategy for AV block, $\mathrm{BBB}$, or high ventricular pacing burden as an alternative to biventricular pacing. The study reported a $90 \%$ success rate with significant QRS narrowing (from $157 \pm 33$ to $117 \pm 18$ $\mathrm{ms}, p<0.001$ ), increase in LVEF (from $30 \pm 10 \%$ to $43 \pm 13 \%$, $p<0.001)$, and improvement in NYHA class (2.8 \pm 0.5 to 1.8 $\pm 0.6, p<0.001$ ) after a mean 14-month follow-up [26]. The authors concluded that consideration should be given to HBP as a rescue strategy for failed biventricular pacing and may be a reasonable primary alternative to biventricular pacing for CRT.

Three randomly controlled trials are ongoing to compare HBP with biventricular CRT (HOPE-HF, His-SYNC

\begin{tabular}{|c|c|c|c|c|c|}
\hline & Barba-Pichardo, et al. & Lustgarden, et al. & Su, et al. & Ajjola, et al. & Sharma ,et al. \\
\hline Total patients & 16 & 29 & 29 & $21(n=16$ for HBP $)$ & 106 \\
\hline Mean age & $68 \pm 6$ & $71(56-87)$ & $65 \pm 11$ & $62 \pm 18$ & $71 \pm 12$ \\
\hline Female (\%) & $32 \%$ & $34 \%$ & $34 \%$ & $25 \%$ & $30 \%$ \\
\hline LVEF prior to implant (\%) & $27 \pm 5$ & $27(21-32)$ & $33 \pm 11$ & $25 \pm 8$ & N/A \\
\hline LVEDD prior to implant (mm) & $66 \pm 4$ & $\mathrm{~N} / \mathrm{A}$ & $65 \pm 9$ & N/A & N/A \\
\hline QRS complex duration prior to implant (ms) & $166 \pm 9 \mathrm{~ms}$ & $169 \pm 16$ & $138 \pm 39$ & $181 \pm 23$ & $157 \pm 33$ \\
\hline LBBB (\%) & $100 \%$ & $97 \%$ & $66 \%$ & 75\% (for HBP) & $34 \%$ \\
\hline Sinus rhythm (\%) & $0 \%$ & $0 \%$ & $61 \%$ & $0 \%$ & $0 \%$ \\
\hline NYHA II/III (\%) & $0 \% / 100 \%$ & $7 \% / 86 \%$ & $53 \% / 47 \%$ & $6 \% / 69 \%$ & N/A \\
\hline Coronary heart disease (\%) & N/A & $55 \%$ & $29 \%$ & $38 \%$ & $51 \%$ \\
\hline Hypertension (\%) & N/A & $57 \%$ & $23 \%$ & N/A & $79 \%$ \\
\hline
\end{tabular}


and NCT02805465). HOPE-HF (The His Optimized Pacing Evaluated for Heart Failure Trial) is currently enrolling with randomized crossover arms for 6 months treatment periods of both - no pacing vs. HBP in patients with heart failure [27]. His-SYNC (His Bundle Pacing Versus Coronary Sinus Pacing for Cardiac Resynchronization Therapy) is another randomized single-blinded study comparing HBP with coronary sinus pacing for CRT in HF patients eligible for biventricular CRT [28]. In NCT02805465, HF patients are being enrolled for comparing HBP with biventricular pacing for atrial fibrillation requiring AV nodal ablation in a randomized, crossover, double- blinded fashion [29].

\section{Proposed mechanisms of LBBB recruitment with HBP}

Multiple potential mechanisms for the recruitment of bundle branches in patients with bundle branch block/delay have been proposed including:

1. longitudinal dissociation in the HB with pacing distal to the site of delay/block; and/or

2. differential source-sink relationships during pacing vs intrinsic impulse propagation; and/or

virtual electrode polarization (VEP) effect [30].

The strongest postulated theory is that of longitudinal dissociation within the HB and intrahisian disease is often responsible for $\mathrm{BBB}$ or delay as elucidated before in the anatomy section of this chapter.

\section{Procedural characteristics of HBP compared with biventricular CRT}

The major procedural characteristics comparing the two modalities for CRT include lead dislodgement rates, capture threshold, procedural time and fluoroscopy time. The lead dislodgement rates with HBP have shown a learning curve with improving rates over a period. Currently the rates of dislodgement have been reported to be similar to right ventricular apical lead placement $[20,25,26,31]$.

Compared to right ventricular pacing, HBP is also associated with a higher capture threshold compared due to His bundle being surrounded by fibrous tissue. However, these capture thresholds have been comparable to LV pacing using coronary sinus deployed lead for biventricular pacing [22,31]. Of note, HBP CRT utilizes only the HBP output compared to both right and left ventricular lead outputs in biventricular CRT devices, suggesting that HBP CRT may, in fact, be equal or more energy efficient. Additionally, the capture thresholds have been shown to be stable over long-term allaying the concerns about quicker battery depletion due to higher followup thresholds [31,32]. Similarly, procedure and fluoroscopy times have improved over time, consistent with the learning curve of adopting a new technique and can be comparable with implantation of traditional right ventricular pacing leads $[19,33,34]$.

\section{Role in current therapy}

Cardiac resynchronization therapy with biventricular pacing has been the cornerstone of therapy to reduce cardiovascular morbidity and mortality in patients with LV systolic dysfunction and heart failure. However, challenges remain due to high (up to 30\%) non-responder rates. PHBP may provide a viable alternative in such patients who are either non-responders or are unable to get biventricular CRT due to coronary sinus anatomy variation or scar tissue. PHBP could also be attempted in patients who fail LV lead placement prior to considering surgical epicardial or endocardial LV lead placement. Additionally, HBP may also be considered in patients with cardiomyopathy and underlying RBBB with or without prolonged PR intervals as an option for cardiac resynchronization therapy $[19,35]$. It remains to be determined if the etiology of cardiomyopathy (ischemic versus nonischemic) play any role in success of CRT with PHBP. Despite the current limitations in data, with improvement in devices and refinement of technique, PHBP is an emerging, viable and safe option for CRT in patients with heart failure.

\section{References}

1. Alanis J, Gonzalez H, Lopez E. The electrical activity of the bundle of His. J Physiol. 1958; 142: 127-140.

PubMed: https://www.ncbi.nlm.nih.gov/pmc/articles/PMC1356698/

2. Giraud G, Puech P, Latour H, Hertault J. [Variations of potential connected with the activity of the auriculo-ventricular condution system in man (endocavitary electrocardiographic recording)]. Arch Mal Coeur Vaiss. 1960; 53: 757-776.

PubMed: https://www.ncbi.nlm.nih.gov/pubmed/13705664

3. Kawashima T, Sasaki H. A macroscopic anatomical investigation of atrioventricular bundle locational variation relative to the membranous part of the ventricular septum in elderly human hearts. Surg Radiol Anat. 2005; 27: 206-213.

PubMed: https://www.ncbi.nlm.nih.gov/pubmed/15723154

4. Kronborg MB, Mortensen PT, Poulsen SH, Gerdes JC, Jensen HK, et al. His or para-His pacing preserves left ventricular function in atrioventricular block: a double-blind, randomized, crossover study. Europace. 2014; 16: 1189-1196.

PubMed: https://www.ncbi.nlm.nih.gov/pubmed/24509688

5. James TN, Sherf L. Fine structure of the His bundle. Circulation. 1971; 44: 9-28.

PubMed: https://www.ncbi.nlm.nih.gov/pubmed/5561420

6. Narula OS. Longitudinal dissociation in the His bundle. Bundle branch block due to asynchronous conduction within the His bundle in man. Circulation. 1977; 56: 996-1006.

PubMed: https://www.ncbi.nlm.nih.gov/pubmed/923070

7. El-Sherif N, Amay YLF, Schonfield C, Scherlag BJ, Rosen K, et al. Normalization of bundle branch block patterns by distal His bundle pacing. Clinical and experimental evidence of longitudinal dissociation in the pathologic his bundle. Circulation. 1978; 57: 473-483.

PubMed: https://www.ncbi.nlm.nih.gov/pubmed/624157

8. Young JB, Abraham WT, Smith AL, Leon AR, Lieberman R, et al. Combined cardiac resynchronization and implantable cardioversion defibrillation in advanced chronic heart failure: the MIRACLE ICD Trial. JAMA. 2003; 289: 2685-2694.

PubMed: https://www.ncbi.nlm.nih.gov/pubmed/12771115 
9. Cleland JG, Daubert JC, Erdmann E, Freemantle N, Gras D, et al. The effect of cardiac resynchronization on morbidity and mortality in heart failure. N Engl J Med. 2005; 352: 1539-1549.

PubMed: https://www.ncbi.nlm.nih.gov/pubmed/15753115

10. Abraham WT, Fisher WG, Smith AL, Delurgio DB, Leon AR, et al. Cardiac resynchronization in chronic heart failure. N Engl J Med. 2002; 346 1845-1553.

PubMed: https://www.ncbi.nlm.nih.gov/pubmed/12063368

11. Bristow MR, Saxon LA, Boehmer J, Krueger S, Kass DA, et al. Cardiacresynchronization therapy with or without an implantable defibrillator in advanced chronic heart failure. N Engl J Med. 2004; 350: 2140-2150. PubMed: https://www.ncbi.nlm.nih.gov/pubmed/15152059

12. Yancy CW, Jessup M, Bozkurt B, Butler J, Casey DE, Jr., et al. 2013 ACCF/AHA guideline for the management of heart failure: a report of the American College of Cardiology Foundation/American Heart Association Task Force on Practice Guidelines. J Am Coll Cardiol. 2013; 62: e147-239.

PubMed: https://www.ncbi.nlm.nih.gov/pubmed/23747642

13. Singh JP, Klein HU, Huang DT, Reek S, Kuniss M, et al. Left ventricular lead position and clinical outcome in the multicenter automatic defibrillator implantation trial-cardiac resynchronization therapy (MADIT-CRT) trial. Circulation. 2011; 123: 1159-1166. PubMed: https://www.ncbi.nlm.nih.gov/pubmed/21382893

14. Chung ES, Leon AR, Tavazzi L, Sun JP, Nihoyannopoulos P, et al. Results of the Predictors of Response to CRT (PROSPECT) trial. Circulation. 2008; 117: 2608-2616.

PubMed: https://www.ncbi.nlm.nih.gov/pubmed/18458170

15. Daubert C, Behar N, Martins RP, Mabo P, Leclercq C. Avoiding nonresponders to cardiac resynchronization therapy: a practical guide. Eur Heart J. 2017; 38: 1463-1472

PubMed: https://www.ncbi.nlm.nih.gov/pubmed/27371720

16. Gamble JHP, Herring N, Ginks M, Rajappan K, Bashir $Y$, et al. Procedural Success of Left Ventricular Lead Placement for Cardiac Resynchronization Therapy: A Meta-Analysis. JACC Clin Electrophysiol. 2016; 2: 69-77.

PubMed: https://www.ncbi.nlm.nih.gov/pubmed/29766856

17. Moss AJ, Hall WJ, Cannom DS, Klein H, Brown MW, et al. Cardiacresynchronization therapy for the prevention of heart-failure events. N Engl J Med. 2009; 361: 1329-1338.

PubMed: https://www.ncbi.nlm.nih.gov/pubmed/19723701

18. Scherlag BJ, Kosowsky BD, Damato AN. A technique for ventricular pacing from the His bundle of the intact heart. J Appl Physiol. 1967; 22: 584-587.

PubMed: https://www.ncbi.nlm.nih.gov/pubmed/6020246

19. Sharma PS, Vijayaraman P. His Bundle Pacing Or Biventricular Pacing For Cardiac Resynchronization Therapy In Heart Failure: Discovering New Methods For An Old Problem. J Atr Fibrillation. 2016; 9: 1501. PubMed: https://www.ncbi.nlm.nih.gov/pmc/articles/PMC5673318/

20. Deshmukh P, Casavant DA, Romanyshyn M, Anderson K. Permanent, direct His-bundle pacing: a novel approach to cardiac pacing in patients with normal His-Purkinje activation. Circulation. 2000; 101: 869-877.

PubMed: https://www.ncbi.nlm.nih.gov/pubmed/10694526

21. Barba-Pichardo R, Manovel Sanchez A, Fernandez-Gomez JM, MorinaVazquez $\mathrm{P}$, Venegas-Gamero $\mathrm{J}$, et al. Ventricular resynchronization therapy by direct His-bundle pacing using an internal cardioverter defibrillator. Europace. 2013; 15: 83-88.

PubMed: https://www.ncbi.nlm.nih.gov/pubmed/22933662

22. Lustgarten DL, Crespo EM, Arkhipova-Jenkins I, Lobel R, Winget
$\mathrm{J}$, et al. His-bundle pacing versus biventricular pacing in cardiac resynchronization therapy patients: A crossover design comparison. Heart Rhythm. 2015; 12: 1548-1557.

PubMed: https://www.ncbi.nlm.nih.gov/pubmed/25828601

23. Su L, Xu L, Wu SJ, Huang WJ. Pacing and sensing optimization of permanent His-bundle pacing in cardiac resynchronization therapy/ implantable cardioverter defibrillators patients: value of integrated bipolar configuration. Europace. 2016; 18: 1399-1405.

PubMed: https://www.ncbi.nlm.nih.gov/pubmed/26581403

24. Vijayaraman P DG, Herweg B, Sharma P, Ellenbogen K. Permanent His bundle pacing is an excellent alternative to cardiac resynchronization therapy. Heart Rhythm. 2016;13:S39.

PubMed: https://www.ncbi.nlm.nih.gov/pubmed/26581403

25. Ajijola OA, Upadhyay GA, Macias C, Shivkumar K, Tung R. Permanent His-bundle pacing for cardiac resynchronization therapy: Initial feasibility study in lieu of left ventricular lead. Heart Rhythm. 2017; 14: 1353-1361.

PubMed: https://www.ncbi.nlm.nih.gov/pubmed/28400315

26. Sharma PS, Dandamudi G, Herweg B, Wilson D, Singh R, et al. Permanent His-bundle pacing as an alternative to biventricular pacing for cardiac resynchronization therapy: A multicenter experience. Heart Rhythm. 2018; 15: 413-420.

PubMed: https://www.ncbi.nlm.nih.gov/pubmed/29031929

27. Health. USNIo. The His Optimised Pacing Evaluation for Heart Failure Trial (HOPE-HF). 2018. https://clinicaltrials.gov/ct2/show/ NCT02671903

28. Health USNIo. His bundle pacing versus coronary sinus pacing for cardiac resynchronization therapy (HisSYNC). https://clinicaltrials. gov/ct2/show/NCT02700425

29. Huang W. Comparison of His bundle pacing and bi-ventricular pacing in heart failure with atrial fibrillation. https://clinicaltrials.gov/ct2/ show/NCT02805465

30. Sharma PS, Huizar J, Ellenbogen KA, Tan AY. Recruitment of bundle branches with permanent His bundle pacing in a patient with advanced conduction system disease: What is the mechanism? Heart Rhythm. 2016; 13: 623-625.

PubMed: https://www.ncbi.nlm.nih.gov/pubmed/26801399

31. Sharma PS, Dandamudi G, Naperkowski A, Oren JW, Storm RH, et al. Permanent His-bundle pacing is feasible, safe, and superior to right ventricular pacing in routine clinical practice. Heart Rhythm. 2015; 12 : 305-312.

PubMed: https://www.ncbi.nlm.nih.gov/pubmed/25446158

32. Vijayaraman P, Dandamudi G, Lustgarten D, Ellenbogen KA. Permanent His bundle pacing: Electrophysiological and echocardiographic observations from long-term follow-up. Pacing Clin Electrophysiol. 2017; 40: 883-891.

PubMed: https://www.ncbi.nlm.nih.gov/pubmed/28569391

33. Vijayaraman P, Dandamudi G, Worsnick S, Ellenbogen KA. Acute HisBundle Injury Current during Permanent His-Bundle Pacing Predicts Excellent Pacing Outcomes. Pacing Clin Electrophysiol. 2015; 38: 540-546. PubMed: https://www.ncbi.nlm.nih.gov/pubmed/25588497

34. Huang WA, Wassie MA, Ajijola OA. Permanent His Bundle Pacing for Cardiac Resynchronization. Curr Treat Options Cardiovasc Med. 2018; 20: 23.

PubMed: https://www.ncbi.nlm.nih.gov/pubmed/29508149

35. Ellenbogen KA, Vijayaraman P. His Bundle Pacing: A New Promise in Heart Failure Therapy? JACC Clin Electrophysiol. 2015; 1: 592-595. PubMed: https://www.ncbi.nlm.nih.gov/pubmed/29759413 